\title{
Summary Abstract: Inelastic tunneling characteristics of AIAs/GaAs heterojunction barriers
}

\author{
R. T. Collins, J. Lambe, and T. C. McGill
}

California Institute of Technology, Pasadena, California 91125

R. D. Burnham

Xerox Corporation, Palo Alto, California 94304

(Received 20 October 1983; accepted 8 February 1984)

PACS numbers: 73.40.Lq, 73.40.Gk, 71.38. + i

Several studies of current transport perpendicular to $\mathrm{Al}_{x} \mathrm{Ga}_{1-x}$ As barriers in $\mathrm{GaAs} / \mathrm{Al}_{x} \mathrm{Ga}_{1-x}$ As double heterojunctions have recently been reported. ${ }^{1-3}$ The currents obtained in these measurements were attributed to thermionic emission over the offsest barrier between the GaAs and $\mathrm{Al}_{x} \mathrm{Ga}_{1-x}$ As and tunneling through the barrier. Chang et al. looked at the tunneling behavior of two $\mathrm{Al}_{x} \mathrm{Ga}_{1-x}$ As barriers separated by a $\mathrm{GaAs}$ quantum well. ${ }^{4}$ In this study we report the first observation of inelastic tunneling in electronic transport perpendicular to a single AlAs barrier sandwiched between two GaAs layers. Temperature dependent $I-V$ and second derivative $\left(d^{2} I / d V^{2}\right)$ measurements were made on AlAs/GaAs double heterojunctions. Reproducible structure was seen in the second derivative spectrum indicating the presence of inelastic tunneling currents. A more detailed account of these measurements and results is given in Ref. 5.

The samples used in this study were grown by a metalorganic chemical vapor deposition (MOCVD) technique. An epitaxial layer of GaAs doped with Se was grown on a degenerately doped GaAs substrate. A thin AlAs layer was then grown on top of the GaAs. The thickness of the AlAs layer varied between 60 and $250 \AA$ from wafer to wafer. A final Sedoped GaAs layer was then grown on the AlAs. The dopings in the GaAs layers were typically $1-5 \times 10^{18} \mathrm{~cm}^{-3}$. The AlAs was either doped $n$-type with $\mathrm{Se}$ at approximately $1 \times 10^{18} \mathrm{~cm}^{-3}$ or doped $p$-type with $\mathrm{Mg}$ at about $1 \times 10^{18}$ $\mathrm{cm}^{-3}$. Mesas were defined on the samples using conventional lithography. ( $\mathrm{Au}, \mathrm{Ge}$ )-Ni contacts were fabricated on the mesas, and the resultant devices were mounted on standard transistor headers and wire bonded to provide contacts. Temperature dependent $I-V$ and second derivative spectra were taken on the samples from room temperature to $4.2 \mathrm{~K}$. Second derivative spectra were taken using a second harmonic detection system as described by Lambe. ${ }^{6}$

First, the results of measurements made on samples with $\mathrm{Mg}$-doped AlAs barriers will be described. The $I-V$ curves for these p-type barriers exhibited increasing resistance with decreasing temperature until approximately $77 \mathrm{~K}$ at which point the curves maintained the same shape down to $4.2 \mathrm{~K}$. The resistivity of the samples increased by many orders of magnitude as the width of the AlAs barrier was increased from 60 to $250 \AA$. For the smaller widths the increase in zero-bias resistivity was approximately exponential with width.
A second derivative spectrum taken from a sample with a $60 \AA$, $p$-type AlAs barrier is given in Fig. 1. Two structures are present in both forward and reverse bias. The first, labeled $a$, is at approximately the optical phonon frequencies of GaAs. It is attributed to an electronic density-of-states effect caused by electron-phonon coupling in the GaAs layers. Similar effects have previously been observed in GaAs Schottky barriers. ${ }^{7}$ The peak labeled b occurs at $50 \mathrm{meV}$ and corresponds to the LO phonon frequency of AlAs. This peak is probably caused by the inelastic excitation of optical phonons in the AlAs layer by tunneling electrons. This is the first observation of these effects in the AlAs/GaAs system.

$I-V$ curves for the Se-doped, $n$-type AlAs layers also displayed increasing resistance with decreasing temperature, but, in contrast with the $p$-type layers, the resistance continued to increase until approximately $30 \mathrm{~K}$. Furthermore, the sample resistivity increased by less than an order of magni-

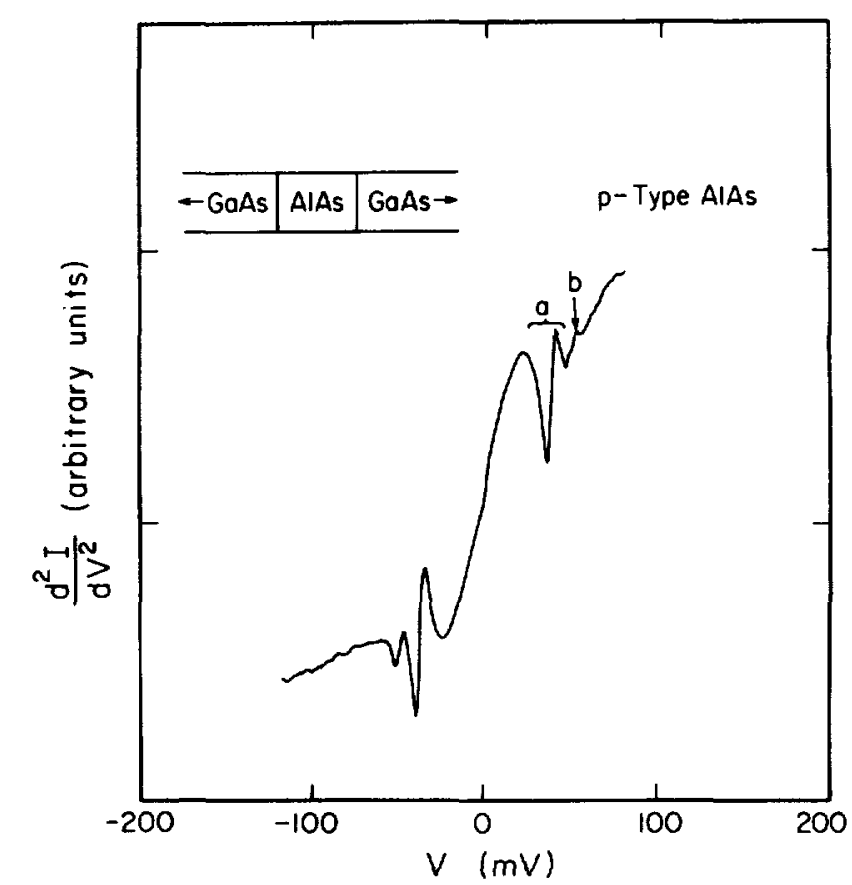

FiG. 1. Second derivative spectrum at $4.2 \mathrm{~K}$ for a p-type AlAs layer sandwiched between two $n$-type GaAs layers. The layer thickness was approximately $60 \AA$. The structure labeled $a$ is attributed to an electronic densityof-states effect caused by electron-optical phonon coupling in the GaAs. The peak labeled $b$ is caused by the inelastic excitation of optical phonons in the AlAs barrier. 


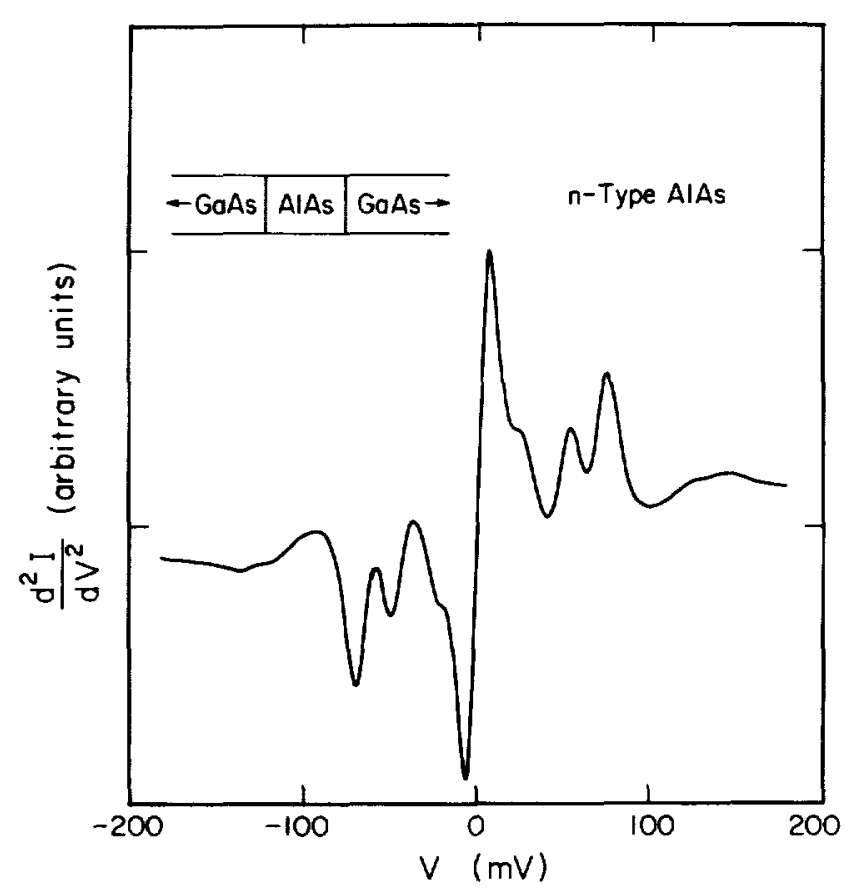

Fig. 2. Second derivative spectrum at $4.2 \mathrm{~K}$ for an $n$-type AlAs layer sandwiched between two $n$-type GaAs layers.

tude as the AlAs layer thicknesses increased from 60 to 250 $\AA$. Changes in slope were visible in the $I-V$ curves. To study these, second derivative spectra were taken at $4.2 \mathrm{~K}$.

Five peaks were visible in both the forward and reverse bias second derivative spectra of the $n$-type barrier samples (Fig. 2). They occurred at approximately $23,50,72,125$, and $145 \mathrm{meV}$. There was also a strong zero-bias anomaly. These peaks did not match those seen in the $p$-type barriers.

A definitive explanation of the results obtained on samples with $n$-type barriers has not been found, but some possible explanations can be proposed. The fact that the $I-V$ curves for the $n$-type barriers froze out at $30 \mathrm{~K}$ suggests that the current flow at $4.2 \mathrm{~K}$ is due to tunneling processes. If transport in the $n$-type AlAs samples is the result of tunneling, the actual tunneling barrier seen by the electrons must not be increasing as the physical width of the AlAs layer is increased. Since doping in the AlAs layer is the critical factor in determining the type of $I-V$ curve and second derivative spectrum obtained from a sample, one possible explanation is that the doping in the $n$-type AlAs layer is actually larger than anticipated, and band bending in the AlAs results in the formation of two back-to-back AlAs/GaAs tunneling barriers with a connecting layer of AlAs. The tunneling resistance of such a structure would be independent of thickness. The dopings given above were estimated from the Se flow rate and may be too low. Then, the peaks in the second derivative spectrum may arise from the excitation of characteristic phonons in the AlAs/GaAs system by tunneling electrons (possibly wave vector-conserving phonons resulting from electrons moving from direct to indirect conduction band minima). A voltage drop would occur across each barrier causing the voltages at which the phonon associated peaks occur to differ from the actual phonon energies. Other explanations for these results include the possibility that more impurities or defects are incorporated into the $n$-type layers than into the $p$-type layer, giving rise to impurity conduction in the barrier. This would not have an exponential dependence on the width of the barrier. The peaks in the second derivative spectrum could indicate the onset of impurity-assisted tunneling or the excitation of vibrational modes of the impurities. Work is currently underway to test these models of current transport in the $n$-type barrier samples.

The authors wish to acknowledge D. L. Smith, C. Mailhiot, T. L. Paoli, and W. Streifer for valuable discussions and are grateful to $H$. Chung, R. D. Yingling, Jr., F. Endicott, M. Bernstein, M. Mosby, J. Tramontana, J. Walker, A. Alimonda, G. L. Harnagel, and R. Ritter for technical assistance with this work. This study was supported in part by the Office of Naval Research under Contract No. N00014-82K-0556.

'D. Delagebeaudeuf, P. Delescluse, P. Etienne, J. Massies, M. Laviron, J. Chaplart, and T. Linh, Electron. Lett. 18, 85 (1982).

${ }^{2}$ A. C. Gossard, W. Brown, C. L. Allyn, and W. Wiegmann, J. Vac. Sci. Technol. 20, 694 (1982).

${ }^{3}$ P. M. Solomon and T. W. Hickmott, Appl. Phys. Lett. 42, 821 (1983).

${ }^{4}$ L. L. Chang, L. Esaki, and R. Tsu, Appl. Phys. Lett. 24, 593 (1974).

${ }^{5}$ R. T. Collins, J. Lambe, T. C. MeGill, and R. D. Burnham, Appl. Phys. Lett. 44, 532 (1984).

'J, Lambe and R. C. Jaklevic, Phys. Rev. 165, 821 (1968).

J. W. Conley and G. D. Mahan, Phys. Rev. 161, 681 (1967). 